ISPRS Annals of the Photogrammetry, Remote Sensing and Spatial Information Sciences, Volume I-7, 2012

XXII ISPRS Congress, 25 August - 01 September 2012, Melbourne, Australia

\title{
HYPERSPECTRAL IMAGE DENOISING WITH CUBIC TOTAL VARIATION MODEL
}

\author{
H. Zhang
}

The State Key Laboratory of Information Engineering in Surveying, Mapping and Remote Sensing, Wuhan University, Wuhan, Hubei, China, zhanghongyan@whu.edu.cn

\section{Commission VII, WG VII/3}

KEY WORDS: Hyperspectral Image, Denoising, Cubic Total Variation, Augmented Lagrangian Method

\begin{abstract}
:
Image noise is generated unavoidably in the hyperspectral image acquision process and has a negative effect on subsequent image analysis. Therefore, it is necessary to perform image denoising for hyperspectral images. This paper proposes a cubic total variation (CTV) model by combining the 2-D total variation model for spatial domain with the 1-D total variation model for spectral domain, and then applies the termed CTV model to hyperspectral image denoising. The augmented Lagrangian method is utilized to improve the speed of solution of the desired hyperspectral image. The experimental results suggest that the proposed method can achieve competitive image quality.
\end{abstract}

\section{INTRODUCTION}

Hyperspectral remote sensing image acquision is a complicated process, in which image noise is generated unavoidably. Hyperspectral image noise has a negative effect on subsequent image processing and analysis, such as classification, target detection, unmixing and etc. Therefore, hyperspectral image denoising aims at removing the noise included in hyperspectral images and supporting improved image analysis capabilities.

To date, many hyperspectral remote sensing image denoising algorithms have been proposed, such as [1-6]. Othman and Qian [1] proposed a hybrid spatial-spectral derivative-domain wavelet shrinkage noise reduction approach. Chen and Qian [2, 3] perform simultaneous dimension reduction and hyperspectral image denoising using wavelet shrinking and principal component analysis. A tenser-filter-based hyperspectral image denoising algorithm was proposed by Salah Bourennane et al. [4]. Another type of filter-based hyperspectral image denoising approach using anisotropic diffusion was proposed in [5]. Recently, Chen et al. [6] proposed a new hyperspectral image denoising algorithm by adding a PCA transform before using wavelet shrinkage; first, a PCA transform was implemented on the original hyperspectral image, and then the low-energy PCA output channel was denoised with wavelet shrinkage denoising processes.

However, most of these denoising algorithms deal with hyperspectral image band by band without considering hyperspectral image cube as a whole integrity. It should be noted that hyperspectral image cube exhibits a 3 dimensional (3-D) concept. On one hand, from the spatial point of view, each band image is a 2-D signal. On the other hand, from the spectral point of view, the spectral curvature forms a 1-D signal. Therefore, this paper proposes a cubic total variation (CTV) model based hyperspectral image denoising method to treat the hyperspectal image as a whole 3-D integrity from both the spatial and spectral dimension.

\section{CUBIC TOTAL VARIATION MODEL BASED HYPERSPECTRAL IMAGE DEBOISING}

\subsection{Cubic Total Variation (CTV) Model}

Total variation (TV) model, firstly proposed by Rudin et al. in 1992 [7], has been widely used in image denoising because of its excellent denoising performance and edge-preserving property. The standard TV model for gray-level image looks like 


$$
R(\boldsymbol{v})=\sum_{m=1}^{M} \sqrt{\left(\nabla_{x} \boldsymbol{v}_{m}\right)^{2}+\left(\nabla_{y} \boldsymbol{v}_{m}\right)^{2}}
$$

where $\nabla_{x}$ and $\nabla_{y}$ represent the gradient operators of the horizontal and vertical directions, and $M$ is the total number of pixels in gray-level image $\boldsymbol{v}$.

Now, we consider the total variation model for hyperspectral image. Firstly, from the perspective of the 2-D spatial domain band by band, each band of the hyperspectral image is a 2-D gray-level image signal. Therefore, the most direct TV model of hyperspectral image is to add the standard TV model of each band and we can get the following 2-D TV model:

$$
R(\boldsymbol{u})=\sum_{b=1}^{B} R\left(\boldsymbol{u}_{b,:}\right)
$$

where $\boldsymbol{u}_{b, \text { : }}$ represents the $b$ th band of hyperspectral image $\boldsymbol{u}, \quad B$ is the number of bands of hyperspectral image, and $R\left(\boldsymbol{u}_{b,:}\right)$ represents the standard TV model for the $b$ th band of hyperspectral image:

$$
R\left(\boldsymbol{u}_{b,:}\right)=\sum_{m=1}^{M} \sqrt{\left(\nabla_{x} \boldsymbol{u}_{b, m}\right)^{2}+\left(\nabla_{y} \boldsymbol{u}_{b, m}\right)^{2}}
$$

where $M$ is the total number of pixels in band $b$ of the hyperspectral image.

Secondly, from the perspective of the 1-D spectral domain pixel by pixel, each pixel of the hyperspectral image is a 1-D spectral signal. The 1-D TV model [8] for the $m$ th pixel of the hyperspectral image can be written as:

$$
R\left(\boldsymbol{u}_{:, m}\right)=\sum_{b=1}^{B}\left|\nabla_{z} \boldsymbol{u}_{b, m}\right|
$$

where $\boldsymbol{u}_{:, m}$ is the 1-D spectral signal of the $m$ th pixel of the hyperspectral image, and $\nabla_{z}$ represent the gradient operator of the spectral domain.

It should be noted that the hyperspectral image cube exhibits a 3-D concept. Therefore, it is natural for us to combine the 2-D total variation model for spatial domain with the 1-D total variation model for spectral domain and propose the termed cubic total variation model for the hyperspectral image, which can be written as:

$$
\begin{gathered}
\boldsymbol{R}_{c}(\boldsymbol{u})=\sum_{b=1}^{B} R_{c}\left(\boldsymbol{u}_{b,:}\right) \\
\boldsymbol{R}_{c}\left(\boldsymbol{u}_{b,:}\right)=\sum_{m=1}^{M} \sqrt{\left(\nabla_{x} \boldsymbol{u}_{b, m}\right)^{2}+\left(\nabla_{y} \boldsymbol{u}_{b, m}\right)^{2}+\beta\left(\nabla_{z} \boldsymbol{u}_{b, m}\right)^{2}}
\end{gathered}
$$

where $\beta$ represents the weight of spectral dimension relative spatial domain.

\subsection{CTV based Hyperspectral Image Denoising}

In recent years, the maximum a posteriori (MAP) [9] estimation theory, which inherently includes prior constraints in the form of prior probability density functions, has been attracting attention and enjoying increasing popularity. Based on the MAP estimation theory, the denoising model for a hyperspectral image can be represented as the following constrained least squares problem:

$$
\hat{\boldsymbol{u}}=\arg \min _{\boldsymbol{u}}\|\boldsymbol{g}-\boldsymbol{u}\|_{2}^{2}+\lambda R(\boldsymbol{u})
$$

where $\boldsymbol{g}$ represents the observed hyperspectral image, the term $\|\boldsymbol{g}-\boldsymbol{u}\|_{2}^{2}$ represents the data fidelity between the observed noisy image and the original clean image, and R(u) is the regularization item, which gives a prior model of the original clear hyperspectral image. $\lambda$ is the regularization parameter which controls the relative contribution of data fidelity term $\|\boldsymbol{g}-\boldsymbol{u}\|_{2}^{2}$ and regularization term $R(\boldsymbol{u})$.

We apply the proposed CTV model in (5) to the hyperspectral image denoising framework and obtain the following cost function:

$$
\hat{\boldsymbol{u}}=\arg \min _{\boldsymbol{u}}\|\boldsymbol{g}-\boldsymbol{u}\|_{2}^{2}+\lambda \sum_{b=1}^{B} R_{c}\left(\boldsymbol{u}_{b,:}\right)
$$

The desired hyperspectral image can be solved by optimizing the cost function shown in (6). Because of the high dimension property of hyperspectral images and the non-difference property of the proposed CTV model, how to efficiently resolve the hyperspectral image denoising model is very important. In this paper, the augmented Lagrangian method [10] is utilized to optimize the CTV denoising model.

\section{EXPERIMENTS}

\subsection{Simulation Results}

The hyperspectral image used in this experiment is a remote sensing image of size $307 \times 280$ collected with HYDICE from Washington DC Mall. In the simulated process, zero-mean Gaussian-noise and salt-and-pepper noise was added to the hyperspectral image. The denoising result with 
ISPRS Annals of the Photogrammetry, Remote Sensing and Spatial Information Sciences, Volume I-7, 2012 XXII ISPRS Congress, 25 August - 01 September 2012, Melbourne, Australia

traditional band-by-band TV model is compared as benchmark. The parameters of the proposed method are set as $\beta=12$ and $\lambda=0.063$. Bands 1 and 21 of the original image, noisy image, denoising results with traditional TV model and with CTV model are shown in Fig. 1. The objective evaluations results are shown in Table 1 . It is clearly observed that the proposed hyperspectral image denoising method outperforms the the hyperspectral image denoising method with the traditional band-by-band TV model in terms of both both the quantitative measurements and visual evaluation.

Table 1 Objective evaluations of denoising results

\begin{tabular}{cccc}
\hline & Noisy image & Traditional TV & CTV \\
\hline MSE & 440.73 & 319.39 & 305.54 \\
\hline PSNR & 21.69 & 23.09 & 23.28 \\
\hline
\end{tabular}
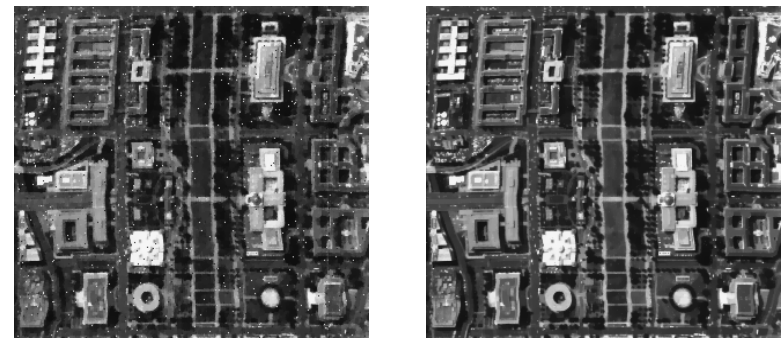

(a) Band 1of the original image, noisy image, denoising results with traditional TV model and with CTV model, respectively
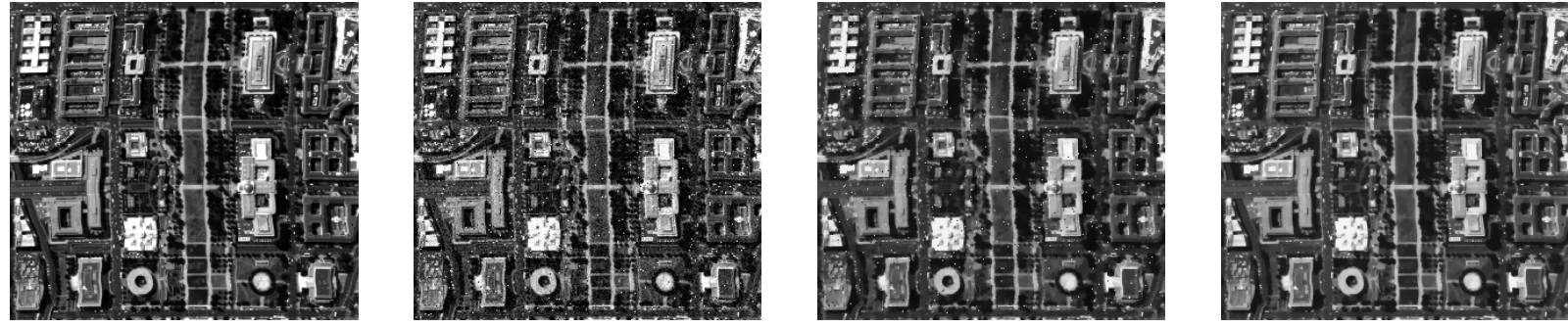

(a) Band 21of the original image, noisy image, denoising results with traditional TV model and with CTV model, respectively

Fig. 1 The denoising result of Washington DC Mall image.

\subsection{Real Results}

The image data used in this experiment is the AVIRIS image of Indian Pine $(145 \times 145$ pixel) acquired in June 1992. The image has 200 bands after discarding water absorption bands. The parameters of the proposed method are set as $\beta=5$ and $\lambda=0.1$. Bands $1,2,3$ and 103 of the original image, noisy image, denoising results with traditional
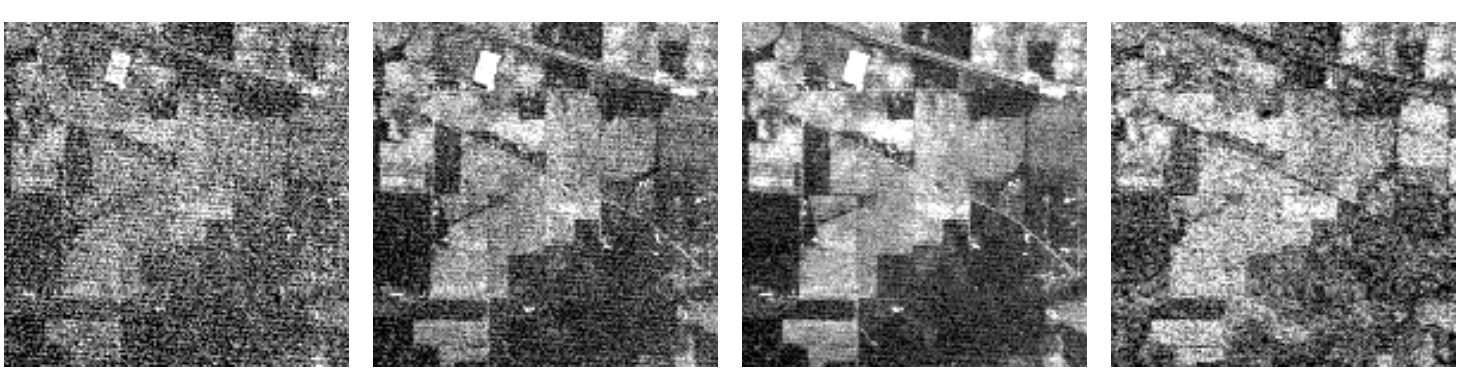

(a) Band 1, 2, 3 and 103 of the original image

TV model and with CTV model are shown in Fig. 2. It is clearly observed that the quality of the denoising results image has improved a lot comparing to the original noisy hyperspectral, and that the hyperspectral image denoising method with the proposed CTV model outperforms the the hyperspectral image denoising method with the traditional band-by-band TV model. 

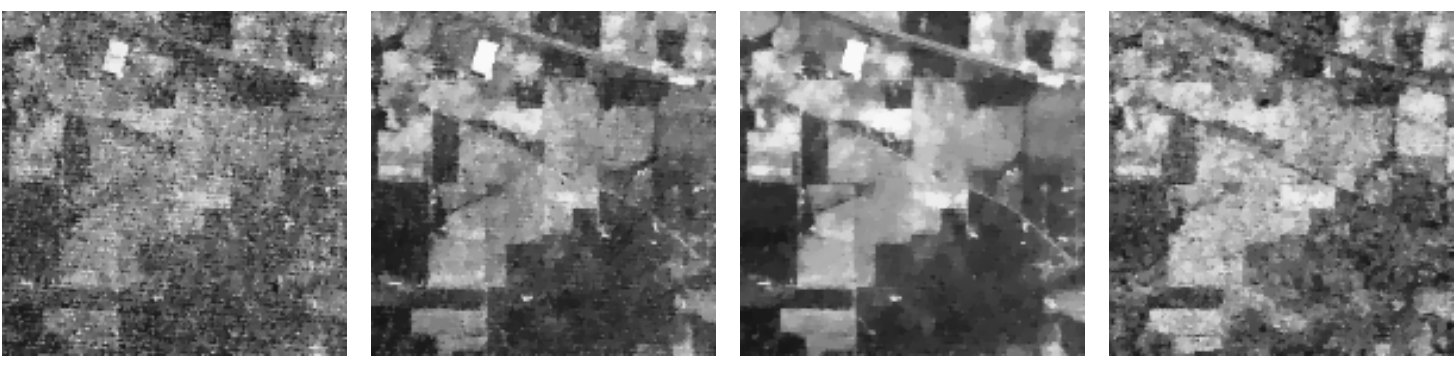

(b) Band 1, 2, 3 and 103 of the denoising results with traditional band-by-band TV model
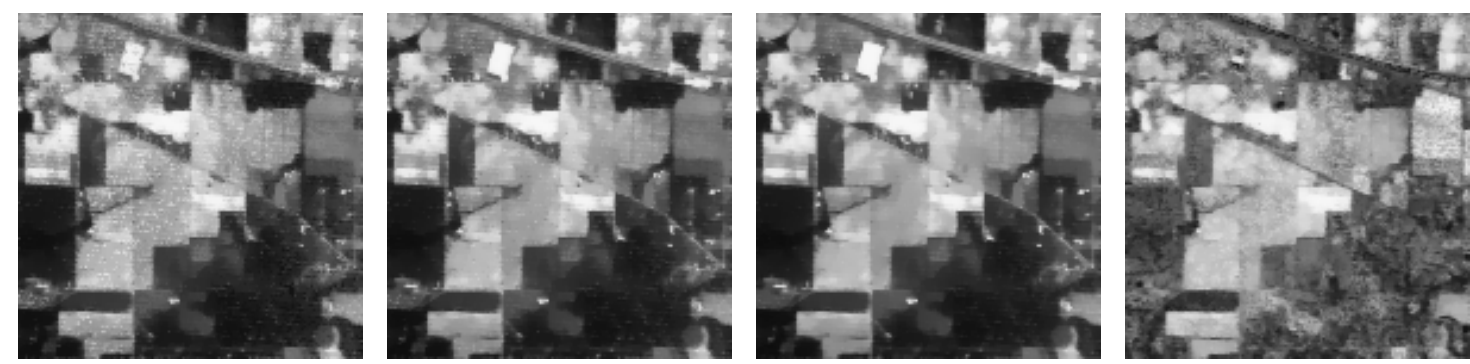

(c) Band 1, 2, 3 and 103 of the denoising results with the proposed CTV model

Fig. 2 The denoising result of Indian image.

\section{CONCLUSIONS}

This paper proposes a cubic total variation model by combining the 2-D total variation model for spatial domain with the 1-D total variation model for spectral domain, and applies the termed cubic total variation model to the hyperspectral image denoising framework. The augmented Lagrangian method is utilized to improve the speed of solution of the desired hyperspectral image. The experimental results suggest that the proposed method can achieve competitive image quality.

\section{ACKNOWLEDGEMENTS}

This work was supported in part by the National Basic Research Program of China (973 Program) under Grant 2011CB707105, by the National Natural Science Foundation of China under Grants 40930532 and 61102112, by Post-doctoral Science Foundation of China under Grant 2011M501242.
[1] Othman H. and Qian S., 2006. Noise reduction of hyperspectral imagery using hybrid spatial-spectral derivative-domain wavelet shrinkage. IEEE Trans. Geosci. Remote Sens., 44(2), pp. 397408.

[2] Chen G. and Qian S., 2008. Simultaneous dimensionality reduction and denoising of hyperspectral imagery using bivariate wavelet shrinking and principal component analysis. Can. J. Remote Sens., 34(5), pp. 447-454.

[3] Chen G. and Qian S., 2009. Denoising and Dimensionality Reduction of Hyperspectral Imagery using Wavelet Packets, Neighbour Shrinking and Principal Component Analysis. Int. J. Remote Sens., 30(18), pp. 4889-4895.

[4] Letexier D. and Bourennane S., 2008. Noise Removal from Hyperspectral Images by Multidimensional Filtering. IEEE Trans. Geosci. Remote Sens., 46(7), pp. 2061-2069.

[5] Wang Y., Niu R., and Yu X., 2010. Anisotropic Diffusion for Hyperspectral Imagery Enhancement. IEEE Sensors J., 10(3), pp. 469-477.

[6] Chen G., Qian S., and Xie W., 2011. Denoising of Hyperspectral Imagery Using Principal Component Analysis and Wavelet Shrinkage. IEEE Trans. Geosci. Remote Sens., 49(3), pp. 973-980.

[7] Rudin L., Osher S., and Fatemi E., 1992. Nonlinear total variation based noise removal algorithms. Physica D, 60(4), pp. 259-268.

[8] Karahanoglu F. I., Bayram I. and De Ville D. V., 2011. A Signal Processing Approach to Generalized 1-D Total Variation. IEEE Trans. Signal Processing, 59(11), pp. 5265-5274.

[9] Zhang L., Zhang H., Shen H., and Li P., 2010. A super-resolution reconstruction algorithm for surveillance images. Signal Process., 90(3), pp. 848-859.

[10] Chan S., Khoshabeh R., Gibson K., Gill P., and Nguyen T., 2011. An Augmented Lagrangian Method for Total Variation Video Restoration. IEEE Trans. Image Process., 20(11), pp. 3097-3111.

\section{REFERENCE}

